\title{
Streptococcal Protein G Enhances Antibody Binding to Platinum Sensor Surfaces
}

\author{
Michael E. Stephens',2, Terri N. Ellis², Jay S. Huebner3, Erica M. Kelly33, Doria F. Bowers ${ }^{2 *}$ \\ ${ }^{1}$ Department of Molecular and Cell Biology, University of Connecticut, Storrs, USA \\ ${ }^{2}$ Department of Biology, University of North Florida, Jacksonville, USA \\ ${ }^{3}$ Department of Physics, University of North Florida, Jacksonville, USA \\ Email: dbowers@unf.edu
}

Received 7 January 2015; accepted 23 January 2015; published 29 January 2015

Copyright (C) 2015 by authors and Scientific Research Publishing Inc.

This work is licensed under the Creative Commons Attribution International License (CC BY).

http://creativecommons.org/licenses/by/4.0/

(c) (i) Open Access

\begin{abstract}
Photo-Electric Microbe Sensor is a patented biotechnology that detects microbes in aqueous solution by measuring the change in photo-voltage in response to UV light stimulation of a platinum (Pt) disk surface on an electrode before and after immunoprecipitation of microbes. This study aimed to increase the sensitivity of microbe detection by pre-adsorbing recombinant Streptococcal Protein G (PG), to the Pt sensor surface. Streptococcal PG binds the Fc region of mammalian IgG molecules and we investigated the association of PG to Pt and the resulting ability to tether antibodies to the Pt-PG surface. An ELISA protocol was optimized to detect the presence of mouse monoclonal antibodies tethered to Pt immunoaffinity disks, and to determine the recommended blocking solution and reagent concentrations. Our results demonstrate that PG binds to bare Pt, increases IgG affinity to the Pt surface following Superblock Buffer application, and together offers design-options for Pt-based sensor technologies.
\end{abstract}

\section{Keywords}

Platinum Sensor Surface, Microbe, Photo-Voltages, Immunoaffinity

\section{Introduction}

Photo-Electric Microbe Sensor (PEMS) has been used to successfully detect a variety of target microbes, such as Sindbis virus, Influenza virus, E. coli and Shigella spp., in ionic aqueous solution [1]. PEMS measures the change observed in induced photo-voltages on the surface of platinum immunoaffinity disks (Pt disk) on electrodes (Figure 1(a)) in response to UV stimulus with and without microbes (Figure 1(b)). These changes in photo-voltage are then correlated to the presence of microbes in aqueous solutions. The eventual aim of this 


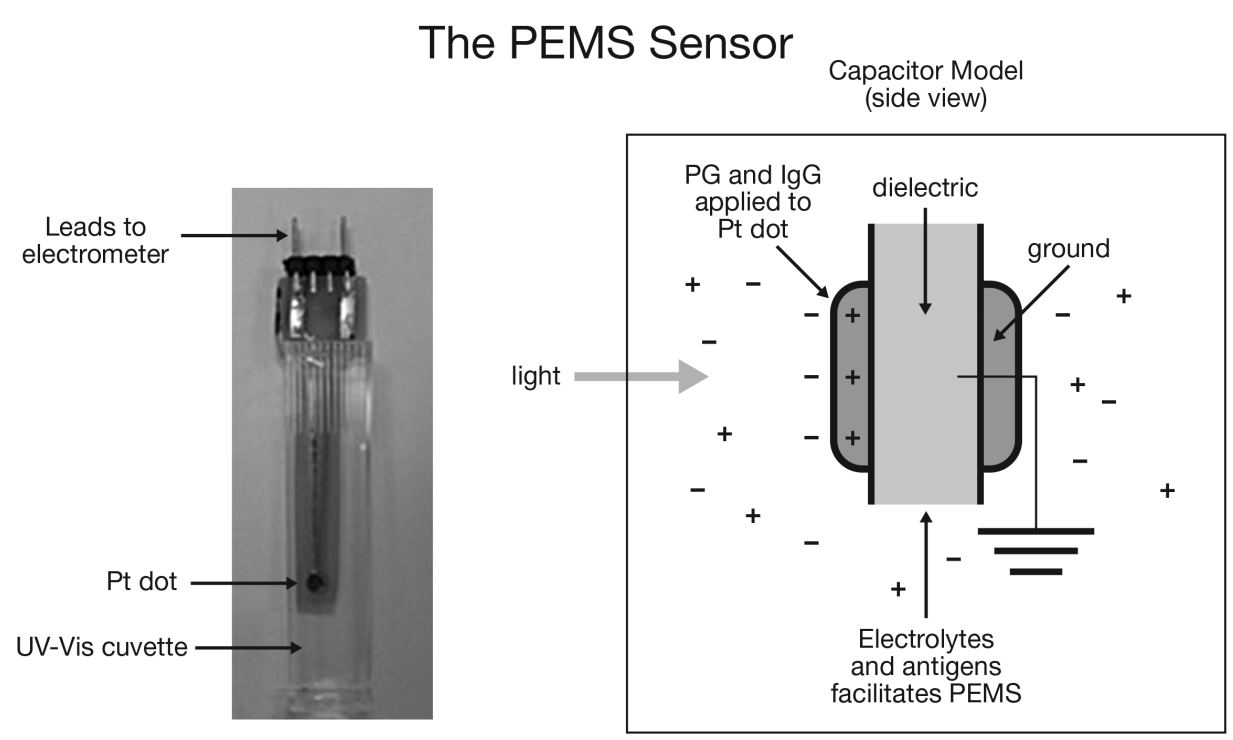

(a)

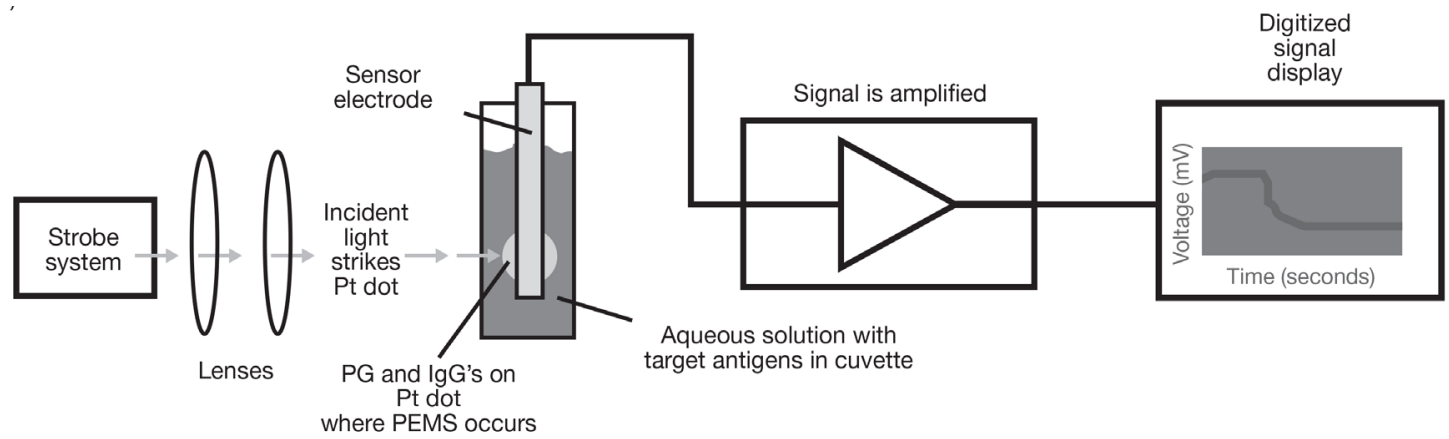

(b)

Figure 1. (a) PEMs electrode: Immunoaffinity Pt disk mounted on dual circuit board electrode prototype. Three arrows indicate the locations of leads to electrometer, the Pt disk providing an available surface (3 mm diameter) for PG and antibody treatment and the UV-Vis cuvette containing solution of target antigens or molecules. The enlarged graphic diagram of electrodes indicates dual Pt surfaces and surrounding charges when submerged in 1 $\mathrm{mM} \mathrm{NaCl}$ test solution. (b) The strobe generates light in the UV spectra which is focused through a series of lenses onto a Pt disk. The subsequent movement of charge produces a photovoltage that is amplified by the electrometer and then displayed on an oscilloscope.

technology is to detect pathogenic agents in environmental waters and biological fluids such as serum, whole blood, and urine. Adsorption of biologicals to Pt surfaces is dependent on a variety of physiochemical conditions [2] and we investigated conditions that would maximize the affinity of binding of antibodies to Pt biosensor surfaces.

Streptococcal protein G (PG) is a surface component of group G Streptococcus cell walls that specifically bind to the Fc domain of IgG antibodies [3]. This binding interaction occurs between PG and all four IgG subclasses [4] and involves mainly charged and polar contacts [5]. The physiologic function of this Fc:PG binding serves to facilitate bacterial infection and avoidance of the host's immune system by nonspecifically coating bacteria with host antibodies [6]. Antibodies bound to PG are oriented with the antigen specific binding pockets facing outwards, away from the pathogen [5] [7]. This ability to specifically bind antibodies aligned in a precise orientation has been utilized to develop antibody-based detection systems [8]-[10]. Studies have also shown that immunoglobulin molecules can be successfully tethered to metals such as gold [10]-[13] and stainless steel [14] by using recombinant PG.

The aim of this study was to enhance the ability of the PEMS system to detect microbes in solution, by utilizing recombinant PG to orient and enhance antibody binding to the platinum electrode. Knowing that recombi- 
nant PG only binds to the Fc domain of IgG molecules we hypothesized that PG will greatly enhance microbe sensor-signaling by lowering detection thresholds, thereby increasing specificity. In order to test this hypothesis an enzyme-linked immunosorbent assay (ELISA) protocol was developed to detect the presence of antibody tethered to the bare Pt disk. Pt disks were pre-treated with recombinant PG then exposed to mouse monoclonal IgG, which was detected using a rabbit anti-mouse IgG horseradish peroxidase conjugate. This study demonstrated that the use of Superblock Blocking Buffer was technically superior to Tween 20 as a blocking agent. Pre-treatment of Pt disk sensor surfaces with PG was efficient and significantly enhanced immunoglobulin binding.

\section{Materials and Methods}

\subsection{Platinum Disk Assemblies and Preparations}

Platinum foil was subjected to a custom punch to make $3 \mathrm{~mm}$ diameter Pt disks with a purity of $99.99 \%$. All disks were cleaned in piranha solution prior to use (3:1 mixture of concentrated Sulfuric Acid, $\mathrm{H}_{2} \mathrm{SO}_{4}$ to $30 \%$ Hydrogen Peroxide, $\mathrm{H}_{2} \mathrm{O}_{2}$ in $\mathrm{H}_{2} \mathrm{O}$ ).

\subsection{Reagents}

Superblock Blocking Buffer in phosphate buffered saline (PBS), recombinant Streptococcal PG, Mouse IgG anti-lipoteichoic acid (LTA), and Rabbit anti-Mouse IgG-horse radish-peroxidase (HRP) conjugate were purchased from Pierce Antibodies, Thermo Scientific. Stabilized Chromogen substrate, TMB, and Stop Solution were purchased from Invitrogen ${ }^{\mathrm{TM}}$.

\subsection{ELISA Assay Protocols}

For ELISA assays, each well in a 96-well plate was blocked using $300 \mu \mathrm{L}$ of either Tween 20 or Superblock following the manufacturer's protocol. All solutions were individually transferred to a new microplate and each absorbance was obtained at $450 \mathrm{~nm}$ on a Universal Microplate Reader (Bio-Tek ELX800). The average absorbance of each group was plotted as a bar graph and the 95\% Confidence Interval (CI; twice the standard deviation) was used to determine statistical significance.

\subsection{ELISA Assay with Tween 20 Block}

For the Tween 20 block, each well was blocked with a 1\% Tween 20 solution in PBS. A single Pt disk was placed in each blocked well containing $60 \mu \mathrm{L}$ of recombinant PG in 10 fold dilutions followed by incubation at $4^{\circ} \mathrm{C}$ overnight $(\mathrm{n}=3)$. Following incubation, wells were washed with $0.05 \%$ Tween 20 in PBS (PBS-T) three times followed by application of Mouse IgG anti-LTA (primary antibody; $0.5 \mu \mathrm{g} / 60 \mu \mathrm{L}$ PBS) incubated at $4^{\circ} \mathrm{C}$ overnight. Wells were then washed and re-blocked as previously described followed by the addition of rabbitanti-mouse HRP (secondary antibody; $0.5 \mu \mathrm{g} / 60 \mu \mathrm{L}$ PBS) and incubated for one hour at room temperature (RT). Wells were washed, TMB substrate was added, signal was allowed to develop, and the reaction was stopped with Stop Solution following the manufacturer's protocol.

\subsection{ELISA Assay with Superblock Buffer}

Alternatively, microplates were blocked with Superblock for one hour at RT, blocking solution was removed and a single pre-cleaned Pt disk was added to each well for a total of five replicates followed by the addition of $100 \mu \mathrm{L}$ of PG 10 -fold dilutions and incubated at $4^{\circ} \mathrm{C}$ overnight. Wells were washed as previously described for a total of three washes and $100 \mu \mathrm{L}$ of primary antibody (mouse IgG anti-LTA in PBS, $1 \mu \mathrm{g} / 100 \mu \mathrm{L}$ ) was added to each well followed by incubation at RT for one hour. Wells were then washed as previously described followed by blocking with Superblock as previously described. Next, $100 \mu \mathrm{l}$ of secondary antibody (rabbit anti-mouse IgG-HRP conjugate) in PBS (1 $\mu \mathrm{g} / 100 \mu \mathrm{L})$ was added to all treatment groups plus an additional negative control blocked well followed by incubation for one hour at RT. The plate was then washed as previously described followed by the addition of $100 \mu \mathrm{L}$ of TMB substrate. The signal was allowed to develop, stopped, and each absorbance was obtained as previously described. 


\section{Results and Discussion}

Platinum surfaces located on PEMS electrodes (Figure 1(a)) detect antigens in solution. Following exposure of sensor to focused flashes of UV light, the signal is converted, amplified, digitized and displayed for analysis (Figure 1(b)). In an effort to enhance antibody binding to metal surfaces of PEMS electrodes, PG was bound to Pt disks by exposure to varying concentrations of recombinant PG.

An ELISA technique was developed to test the binding of mouse anti-LTA IgG by use of a secondary rabbit anti-mouse IgG-HRP conjugate in 96-well plates. Using 1\% Tween 20 in PBS as the well-blocking buffer, only PG at $100 \mu \mathrm{g} / \mathrm{mL}$, the highest concentration used, demonstrated a significant signal over background (Figure 2). In contrast, using Superblock as the well-blocking agent produced three to six fold increases in the absorbance readings and reduced background binding noise of secondary antibody (Figure 3). Using PG (>1.0 mg/mL) or $(250 \mu \mathrm{g} / \mathrm{mL})$ observed at the 95\% C.I. demonstrated significant significance compared to binding using PG (25 $\mu \mathrm{g} / \mathrm{mL}$ ) (Figure 3).

Pre-treating Pt disk surfaces with PG enhanced antibody binding compared with untreated surfaces, especially following Superblock application. We recommend this methodology for binding immunoglobulin molecules to Pt surfaces and found that Tween 20 was not as sufficient a blocking agent as Superblock because of the presence of high background binding of the secondary antibody and lack of significant differences between groups. Given that both of the two highest concentrations of PG resulted in maximum signal, we conclude that PG binding to Pt disc's were saturated at any concentration above $250 \mu \mathrm{g} / \mathrm{ml}$. Groups exposed to PG (25 $\mu \mathrm{g} / \mathrm{mL})$ concentrations and higher were significantly different from other groups at the 95\% confidence interval indicating a decreased antibody binding. We report that the presence of PG molecules and their inherent ability to orient IgG's is reflected in and accounts for the increased binding of immunoglobulins to Pt surfaces (Figure 4).

\section{Conclusion}

This data demonstrates that recombinant streptococcal PG ( $\geq 250 \mu \mathrm{g} / \mathrm{mL})$ can enhance the binding of antibodies to the surface of Pt disks following Superblock Buffer application. Suggested future studies include testing the immunoaffinity properties of Pt immunoaffinity disks in an ELISA application, challenging the detection capability using PEMS testing, as well as the ability of PG to interact/bind with other metals without the use of SAMs. We propose that pre-adsorption of PG to Pt in conjunction with Superblock Buffer will enhance the binding of immunoglobulins to the Pt sensor surface, leading to new sensor technologies or enhancement of existing ones.

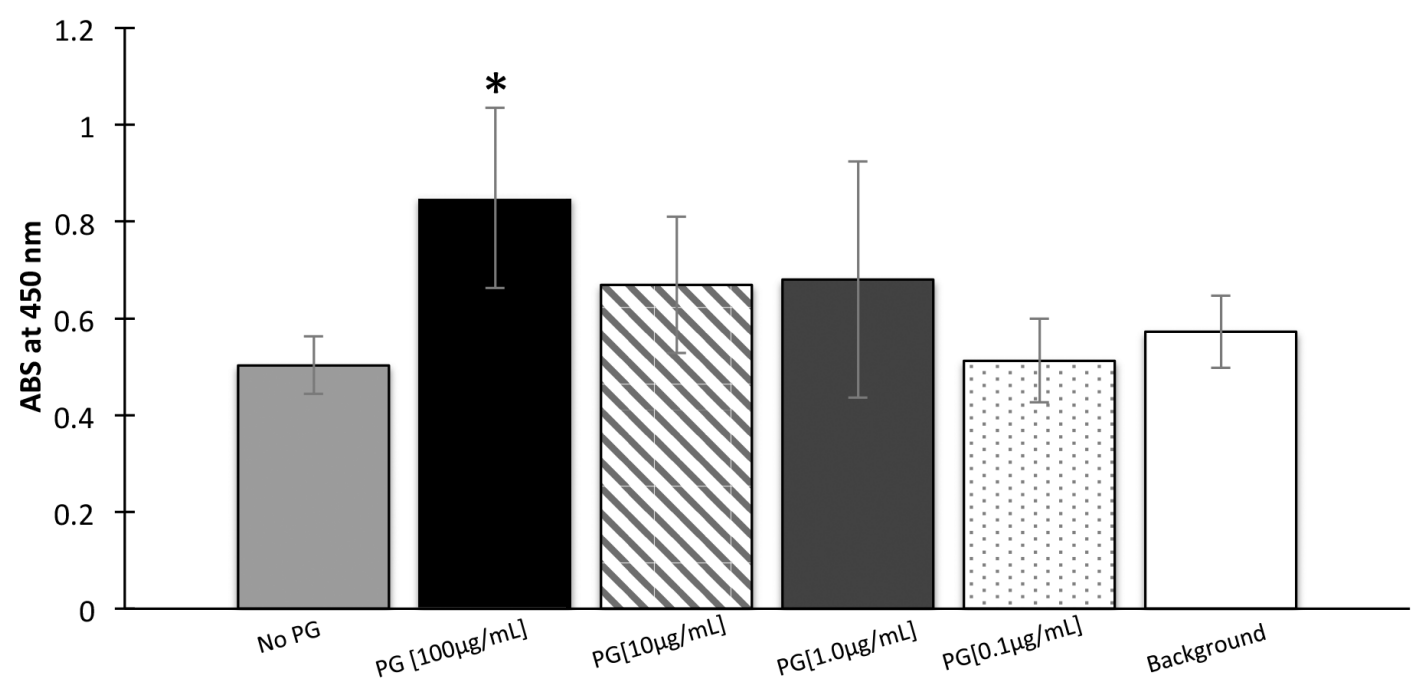

Figure 2. Effects of recombinant PG on antibody binding to Pt disks using 1\% Tween 20 in PBS as a blocking agent. Pre-cleaned Pt disk were exposed to varying concentrations of PG in blocked wells (using 1\% Tween 20 buffer), next exposed to the primary $\mathrm{AB}$ (mouse IgG anti-LTA), followed by incubation in the secondary $\mathrm{AB}$ (rabbit anti-mouse IgG). After development of the chromogen, average absorbance of each reaction was documented and error bars represent the 95\% Confidence Interval. Statistical significance indicated by *. 


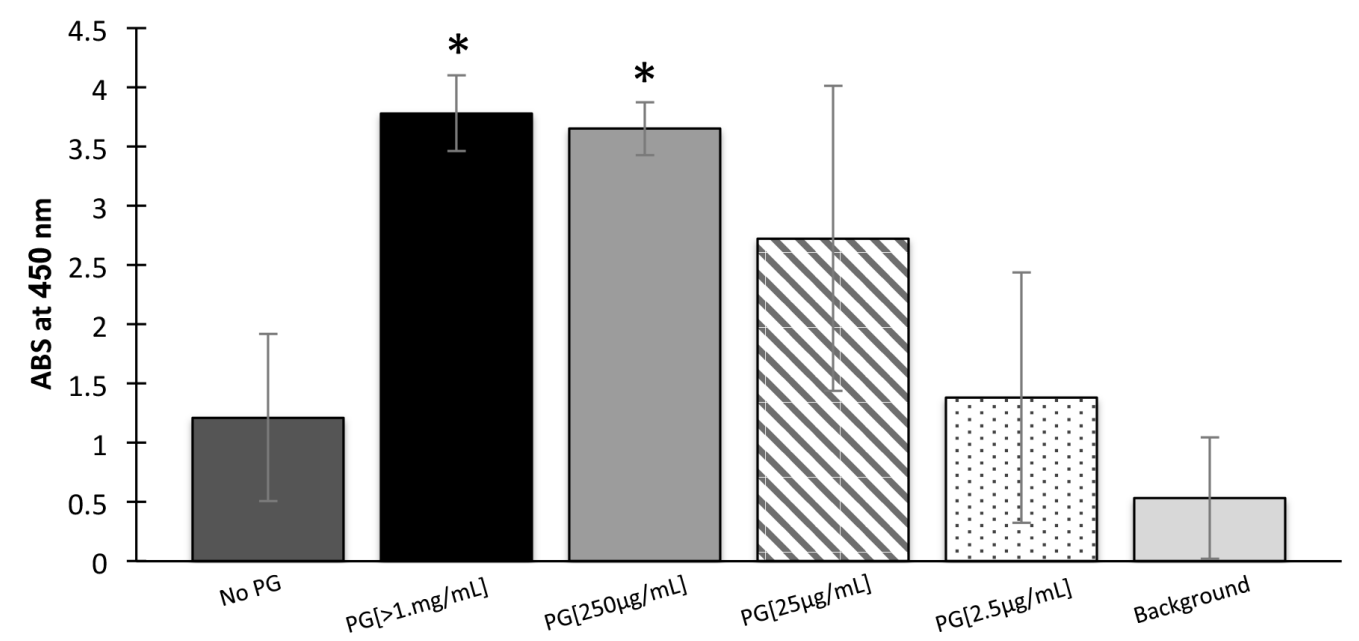

Figure 3. Effects of recombinant PG on antibody binding to Pt disks using Superblock in PBS as a blocking agent. Pre-cleaned Pt disk were exposed to varying concentrations of PG (at a 1:10 dilution) in each blocked well (using Superblock), next exposed to the primary AB (mouse IgG anti-LTA), followed by incubation in the secondary AB (rabbit anti-mouse IgG). After development of the chromogen, average absorbance of each reaction was documented and error bars represent the 95\% Confidence Interval. Statistical significance indicated by *.

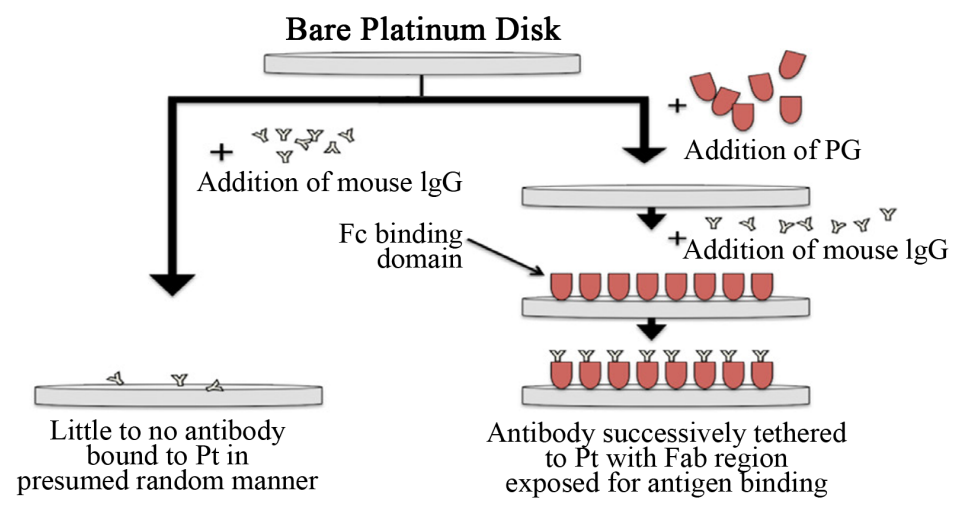

Figure 4. Visual representation comparing the tethering of antibody (mouse IgG) to platinum disk electrode surface, comparing absence with presence of recombinant PG. Pre-adsorption of PG to Pt sensor surfaces enhances the specific binding of the Fc region of mammalian IgG [3]. This oriented attachment of antibody [5] [7] serves to expose the Fab region, which maximizes the tethering capability for immunoprecipitation of antigens.

\section{Acknowledgements}

This work was partially supported by DOD grant W911SR-07-C-0099. We thank Michael Boyles (Center for Instruction and Research Technology-UNF) for his expertise in computer graphics.

\section{References}

[1] Huebner, J.S., Mejia, E.N. and Bowers, D.F. (2011) Sensing Device and Method for Rapidly Determining Concentrations of Microbial Organisms Using Interfacial Photo-Voltages. The US Patent Office Issued Patent Number 7892495.

[2] Cosman, N.P. and Roscoe, S.G. (2004) Electrochemical Quartz Crystal Nanobalance (EQCN) Studies of Protein Interfacial Behavior at Pt. Langmuir, 20, 1711-1720. http://dx.doi.org/10.1021/la035154h

[3] Myhre, E.B. and Kronvall, G. (1977) Heterogeneity of Nonimmune Immunoglobulin Fc Reactivity among Gram-Positive Cocci: Descriptions of Three Major Types of Receptors for Human Immunoglobulin G. Infection and Immunity, 17, 475-482.

[4] Björck, I. and Kronvall, G. (1984) Purification and Some Properties of Streptococcal Protein G, a Novel IgG-Binding 
Reagent. J. Immunol., 133, 969-974.

[5] Sauer-Eriksson, A.E., Klewegt, F.J., Uhlen, M. and Jones, T.A. (1995) Crystal Structure of the C2 Fragment of Streptococcal Protein G in Complex with the Fc Domain of Human IgG. Structure, 3, 265-278. http://dx.doi.org/10.1016/S0969-2126(01)00157-5

[6] Gronenborn, A., Filpula, D., Essig, N., Achari, A., Whitlow, M., Wingfield, P. and Clore, G. (1991) A Novel, Highly Stable Fold of the Immunoglobulin Binding Domain of Streptococcal Protein G. Science, 253, 657-661. http://dx.doi.org/10.1126/science.1871600

[7] Nordenfelt, P., Waldemarson, S., Linder, A., Mörgelin, M., Karlsson, C., Malmström, J. and Björck, L. (2012) Antibody Orientation at Bacterial Surfaces Is Related to Invasion Infection. Journal of Experimental Medicine, 209, 23672381. http://dx.doi.org/10.1084/jem.20120325

[8] Camarero, J.A. (2007) Recent Developments in the Site-Specific Immobilization of Proteins onto Solid Supports. Biopolymers (Peptide Sci.), 90, 450-458.

[9] Rusmini, F., Zhong, Z. and Feijen, J. (2007) Protein Immobilization Strategies for Protein Biochips. Biomacromolecules, 8, 1775-1789. http://dx.doi.org/10.1021/bm061197b

[10] Niu, Y., Matos, A.I., Abrantes, L.M., Viana, A.S. and Jin, G. (2012) Antibody Orientated Immobilization on Gold Using the Reaction between Carbon Disulfide and Amine Groups and Its Application in Immunosensing. Langmuir, 28, 17718-17725. http://dx.doi.org/10.1021/la303032f

[11] Nuebert, H., Jacoby, E.,Bansai, S., Lies, R., Cowan, D. and Kicman, A. (2002) Enhanced Affinity Capture MALDITOF MS: Orientation of an Immunoglobulin G Using Recombinant Protein G. Analytical Chemistry, 74, 3677-3683. http://dx.doi.org/10.1021/ac025558z

[12] Wanabe, H., Kanazake, K., Nakanishi, T., Shiotsuka, H., Hatakeyama, H., Kaieda, H., Imamura, T., Umetsu, M. and Kumagai, I. (2011) Biomimetic Engineering of Modular Bispecific Antibodies for Biomolecule Immobilization. Langmuir, 16, 9656-9661. http://dx.doi.org/10.1021/la2006259

[13] Zhang J., Sun, Y., Xu, B., Zhang, H., Gao, Y., Zhang, H. and Song, D. (2013) A Novel Surface Plasmon Resonance Biosensor Based on Grapheme Oxide Decorated with Gold Nanorod-Antibody Conjugates for Determination of Transferrin. Biosensors and Bioelectronics, 45, 230-236. http://dx.doi.org/10.1016/j.bios.2013.02.008

[14] Hedberg, Y., Wang, X., Hedberg, J., Lundin, M., Blomberg, E. and Wallinder, I.O. (2013) Surface-Protein Interactions on Different Stainless Steel Grades: Effects of Protein Adsorption, Surface Changes and Metal Release. Journal of Materials Science: Materials in Medicine, 24, 1015-1033. http://dx.doi.org/10.1007/s10856-013-4859-8 
Scientific Research Publishing (SCIRP) is one of the largest Open Access journal publishers. It is currently publishing more than 200 open access, online, peer-reviewed journals covering a wide range of academic disciplines. SCIRP serves the worldwide academic communities and contributes to the progress and application of science with its publication.

Other selected journals from SCIRP are listed as below. Submit your manuscript to us via either submit@scirp.org or Online Submission Portal.
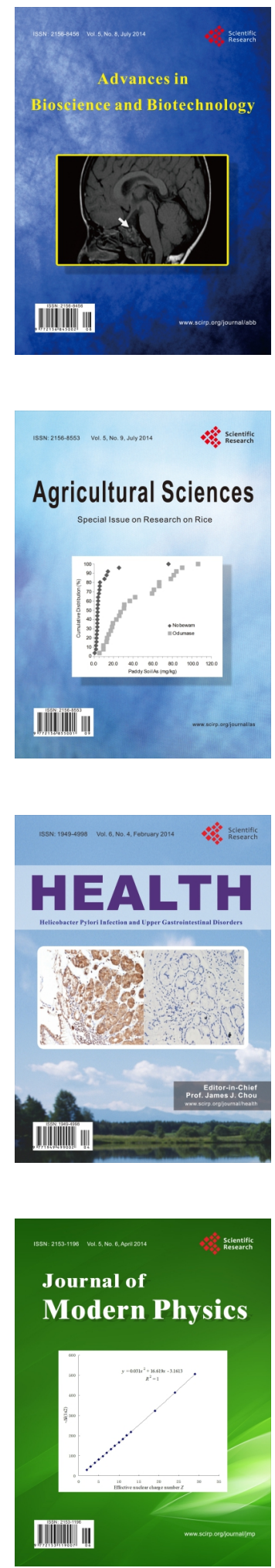
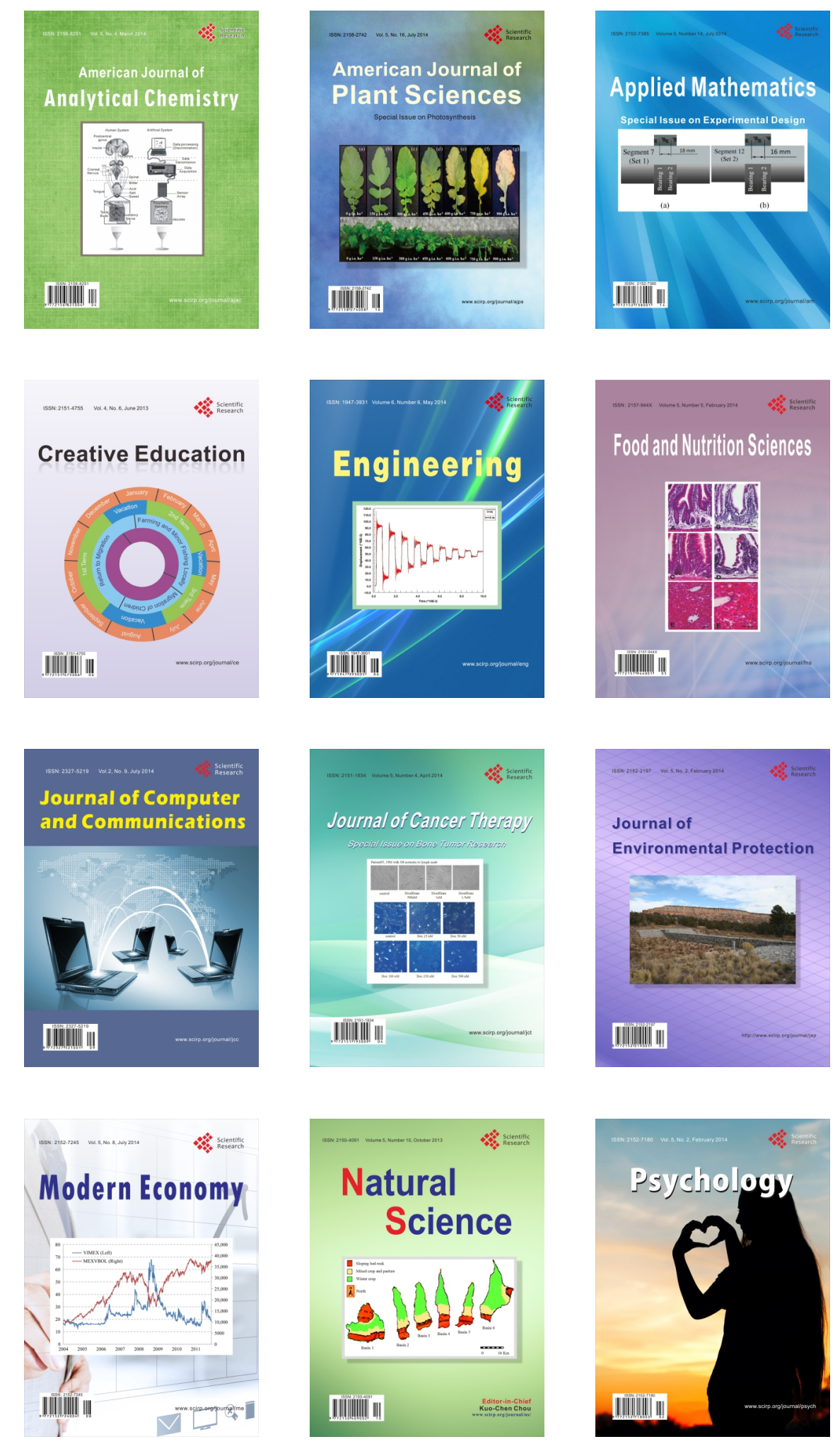